\title{
Smartphone assisted naked eye detection of mercury (II) ion using horseradish peroxidase inhibitive assays
}

\begin{abstract}
Background: Mercury (II) ion, $\mathrm{Hg} 2+$ is among the most common pollutants with the ability to affect the environment. The implications of their elevation in the environment are mainly due to the industrialization and urbanization process. Current methods of $\mathrm{Hg} 2+$ detection primarily depend on sophisticated and expensive instruments. Hence, an alternative and practical way of detecting $\mathrm{Hg} 2+$ ions is needed to go beyond these limitations. Here, we report a detection method that was developed using an inhibitive enzymatic reaction that can be monitored through a smartphone. Horseradish peroxidase (HRP) converted 4-aminoantipyrene (4-AAP) into a red colored product which visible with naked eye. A colorless product, on the other hand, was produced indicating the presence of $\mathrm{Hg} 2+$ that inhibit the reaction. Objectives: The aim of this study is to develop a colorimetric sensor to detect $\mathrm{Hg} 2+$ in water sources using HRP inhibitive assay. The system can be incorporated with a mobile app to make it practical for a prompt in-situ analysis. Methods: HRP enzyme was pre-incubated with different concentration of $\mathrm{Hg} 2+$ at $37^{\circ} \mathrm{C}$ for 1 hour prior to the addition of chromogen. The mix of PBS buffer, 4-AAP and phenol which act as a chromogen was then added to the HRP enzyme and was incubated for 20 minutes. Alcohol was added to stop the enzymatic reaction, and the change of colour were observed and analyse using UV-Vis spectrophotometer at $520 \mathrm{~nm}$ wavelength. The results were then analysed using GraphPad PRISM 4 for a non-linear regression analysis, and using Mathematica (Wolfram) 10.0 software for a hierarchical cluster analysis. The samples from spectroscopy measurement were directly used for dynamic light scattering (DLS) evaluation to evaluate the changes in HRP size due to $\mathrm{Hg} 2+$ malfunctionation. Finally, molecular dynamic simulations comparing normal and malfunctioned HRP were carried out to investigate structural changes of the HRP using YASARA software. Results: Naked eye detection and data from UV-Vis spectroscopy showed good selectivity of $\mathrm{Hg} 2+$ over other metal ions as a distinctive color of $\mathrm{Hg} 2+$ is observed at $0.5 \mathrm{ppm}$ with the IC50 of $0.290 \mathrm{ppm}$. The mechanism of $\mathrm{Hg} 2+$ inhibition towards HRP was further validated using a dynamic light scattering (DLS) and molecular dynamics (MD) simulation to ensure that there is a conformational change in HRP size due to the presence of $\mathrm{Hg} 2+$ ions. The naked eye detection can be quantitatively determined using a smartphone app namely ColorAssist, suggesting that the detection signal does not require expensive instruments to be quantified. Conclusion: A naked-eye colorimetric sensor for mercury ions detection was developed. The colour change due to the presence of $\mathrm{Hg} 2+$ can be easily distinguished using an app via a smartphone. Thus, without resorting to any expensive instruments that are mostly laboratory bound, $\mathrm{Hg} 2+$ can be easily detected at IC50 value of $0.29 \mathrm{ppm}$. This is a promising alternative and practical method to detect $\mathrm{Hg} 2+$ in the environment.
\end{abstract}

Keyword: Horseradish peroxidase; Mercury detection; Colorimetric assay; Inhibitive assay; Naked eye detection; Smartphone 